\title{
Pseudo-stroke manifestation of multiple myeloma: a report of two cases with literature review
}

\author{
Piotr Czapiewski ${ }^{1}$, Tomasz Szmuda², Jarosław Dzierżanowski², Paweł Słoniewski², Krzysztof Sworczak ${ }^{3}$, Maria Gnacińska³ \\ Mirosława Dubaniewicz-Wybieralska ${ }^{4}$, Andrzej Hellmann ${ }^{5}$, Wojciech Biernat ${ }^{1}$ \\ ${ }^{1}$ Department of Pathology, ${ }^{2}$ Department of Neurosurgery, ${ }^{3}$ Department of Endocrinology and Internal Medicine, ${ }^{4}$ Department of Radi- \\ ology, ${ }^{5}$ Department of Haematology and Transfusiology, Medical University of Gdansk, Gdansk, Poland
}

\begin{abstract}
Multiple myeloma rarely presents with neurological symptoms. We describe two patients with pseudo-stroke manifestation of multiple myeloma. The first patient was a 60-year-old female with the initial presentation of multiple myeloma forming a large skull tumour compressing the brain with resultant symptoms of aphasia, limited logical contact and right upper extremity paresis. The second patient was a 69-year-old female who presented with a partial motor epileptic seizure involving both right limbs with persisting weakness of muscles and speech disturbances due to a skull tumour 4 years after the diagnosis of multiple myeloma. Pseudo-stroke manifestation of multiple myeloma is extremely uncommon, however it can sometimes be observed both as an initial presentation and in the course of long-lasting disease.
\end{abstract}

Key words: multiple myeloma, stroke, aphasia, hemiparesis, aspiration biopsy.

\section{Introduction}

Multiple myeloma (MM) is a relatively frequent plasma cell neoplasm. Clinical symptoms dependent on bone marrow myeloma expansion are associated with skeletal destruction, infection and renal failure. However, other clinical symptoms may also occur, including neurological symptoms, which are usually a result of hypercalcemia or compression of the spinal column. Occasionally, an apoplectic manifestation of MM has been reported. The following is a report on the MM history in two female patients; one of them developed a skull tumour that compressed the cerebrum and resulted in hemiparesis and aphasia as the initial pre- sentation of the disease. The other patient developed very similar symptoms, however, they appeared at the end stage of the disease.

\section{Patient 1}

A 60-year-old female was admitted to the emergency unit with aphasia, limited logical contact and right upper extremity paresis that appeared the previous day. Gradual deterioration in speech, difficulties in constructing sentences and impeded comprehension were present for the last seven months.

On admission, the patient showed vestigial logical contact. The neurological examination showed comple- 
Piotr Czapiewski, Tomasz Szmuda, Jarosław Dzierżanowski, Paweł Słoniewski, Krzysztof Sworczak, Maria Gnacińska,

Mirosława Dubaniewicz-Wybieralska, Andrzej Hellmann, Wojciech Biernat

te motor and partial sensory aphasia with right-sided hemiparesis. In addition, a tumour in the left frontoparietal region of the skull was found by palpation. On CT examination, the tumour was located in the cranial vault infiltrating the overlying subcutaneous tissue as well as the adjacent cranial cavity, compressing the frontal and the parietal lobe (Fig. 1). A stereotactic biopsy was performed and the diagnosis of plasma cell neoplasm was rendered (Fig. 2). It was confirmed by immunostaining with CD138 (Fig. 3) and CD56 (Fig. 4).

Bone marrow aspiration cytology showed features of megaloblastic anaemia and the presence of plasmacytoid cells. Multiple myeloma was suspected and subsequently confirmed by an elevated IgG serum lev-

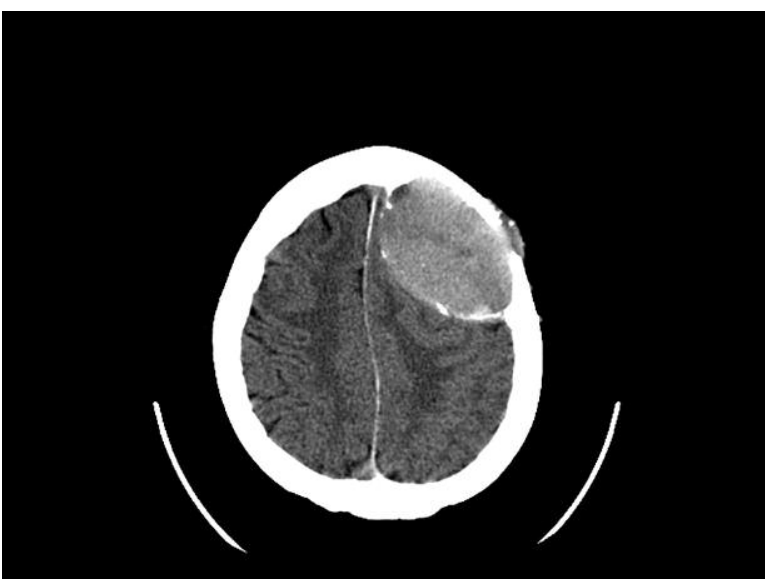

Fig. 1. Tumour located in the bone of the skull vault, infiltrating the overlying subcutaneous tissue and adjacent skull cavity, compressing the frontal and the parietal lobe.

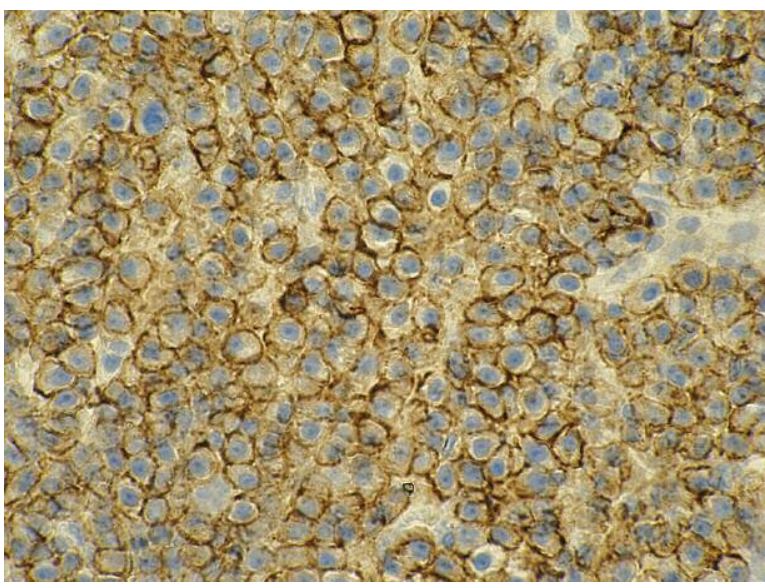

Fig. 3. Strong membranous reaction with antiCD138 antibody. el and a strong monoclonal IgG kappa band (monoclonal band $-42 \%)$. Whole body radiography revealed osteolytic lesions in the parietal bone and the ribs. Radiotherapy to the left frontoparietal region of the skull (20 Gy/5 fraction) and three courses of dexamethasone (40 mg/day) were administered. The treatment resulted in improvement of laboratory parameters and neurological condition. Paresis and aphasia subsided, but the patient still presented symptoms of the frontal lobe syndrome. She was transferred to the Department of Haematology for further treatment.

\section{Patient 2}

In June 2003, a 69-year-old female was admitted to a municipal hospital for a clinical work-up of a pro-

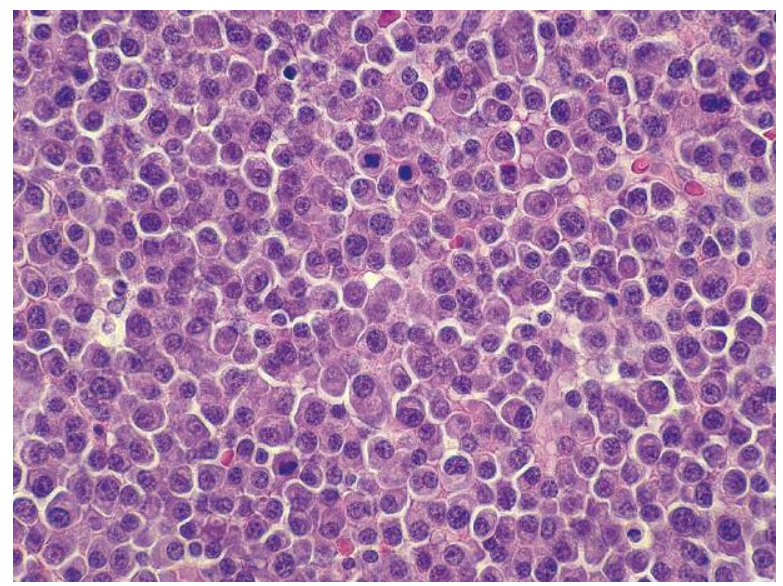

Fig. 2. Tightly arranged neoplastic plasma cells with abundant eosinophilic cytoplasm and eccentric nuclei.

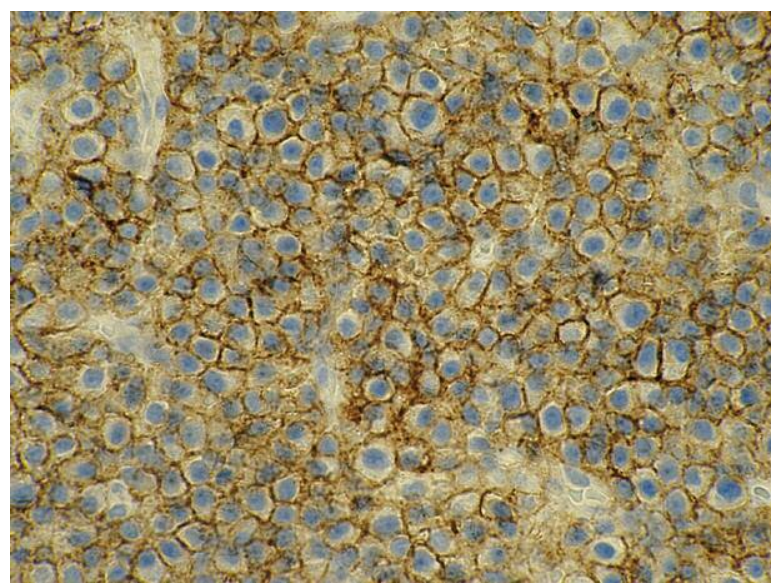

Fig. 4. Strong expression of CD56. 


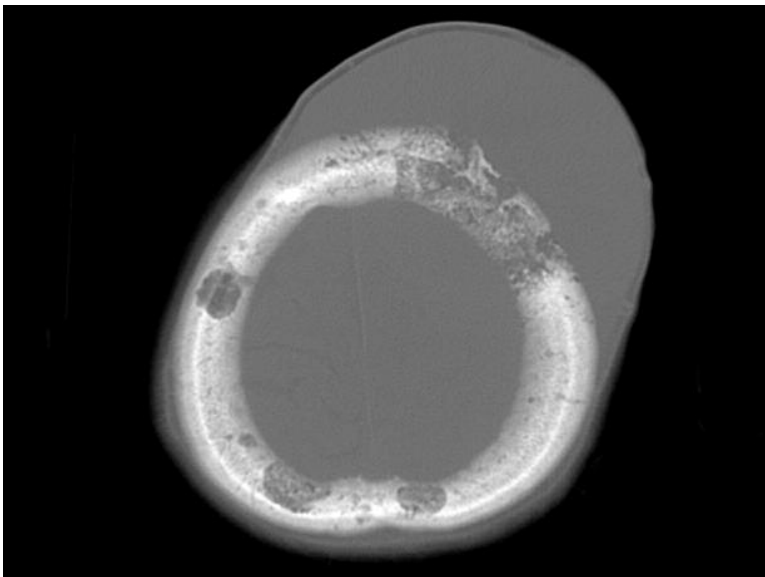

Fig. 5. Pathological mass compressing frontal and parietal lobe with osteolytic foci in the bone.

gressively worsening anaemia. The myelogram showed an increase in the number of plasma cells (ca. 30\% of nucleated bone marrow elements). A monoclonal peak in the gamma globulin fraction of the blood plasma proteins was also revealed. Upon X-ray examination, multiple osteolytic foci in the skull were found, with the largest one in the left parietal bone $(50 \mathrm{~mm}$ in diameter), iliac crest, pelvic bones and proximal femur, as well as features of generalized osteoporosis.

The patient was initially treated with 3 courses of VMCP/VBAP and 6 courses of dexamethasone with a partial clinical response, followed by 4 courses of BCD. A long-standing decrease in the number of plasma cells in the bone marrow was noted. Her clinical picture was uneventful except for two episodes of pneumonia.

In July 2007, the patient was admitted to the Department of Neurology due to a partial motor epileptic seizure involving both right limbs with persisting muscle weakness and speech disturbances. On physical examination, a palpable mass in the left frontoparietal area was found. On neurological examination, the right pyramidal syndrome was identified, with a high degree of hemiparesis and sensorimotor aphasia.

Upon CT investigation, multiple osteolytic lesions in the skullcap were found, the largest one was located in the frontoparietal region, with a diameter of $60 \mathrm{~mm}$. In the vicinity of the largest osteolytic lesion in the left cranial vault, a hyperintensive pathological structure $100 \times 80 \mathrm{~mm}$ in size was found showing contrast enhancement. The larger portion of the lesion was located extracranially, the smaller one (50 x $32 \mathrm{~mm})$ - intracranially. The latter exerted mass effect on the cortical sulci of the left brain hemisphere, left lateral

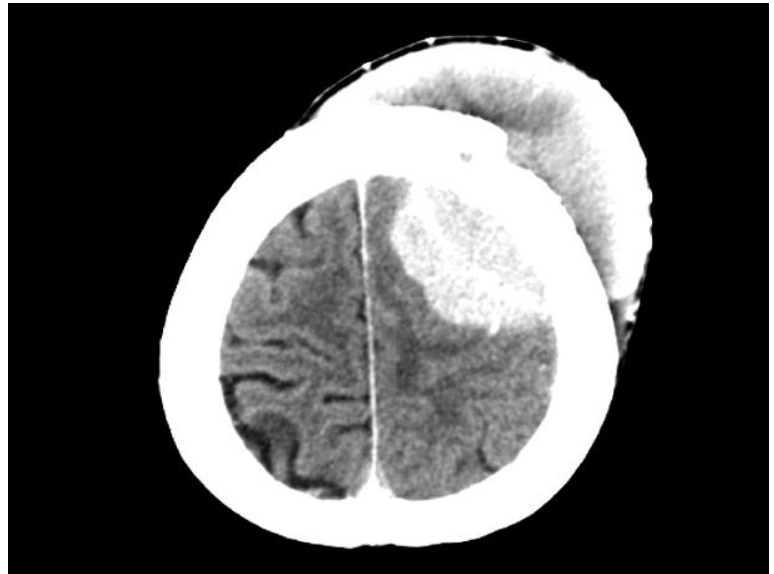

Fig. 6. Strong contrast enhancement of the lesion.

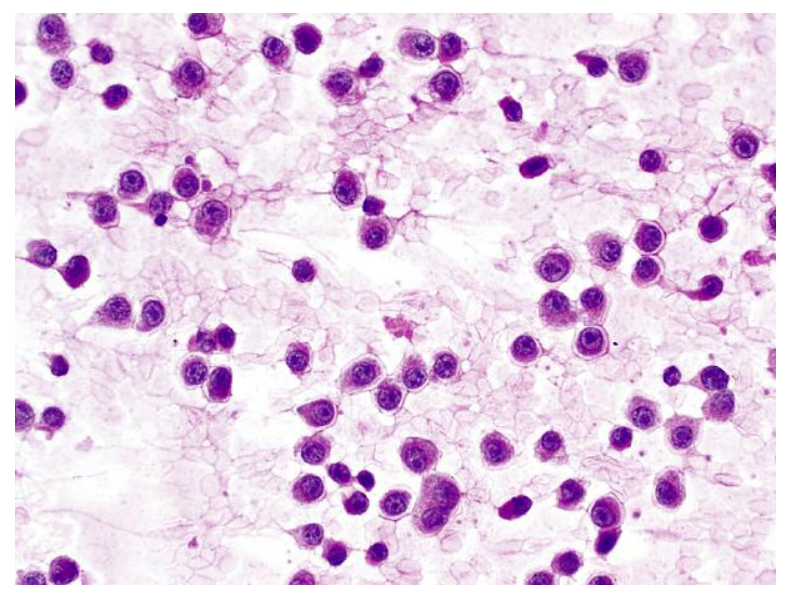

Fig. 7. Multiple plasma cells in aspirate from the skull tumour of patient 2.

ventricle with a shift of the median structures $6 \mathrm{~mm}$ to the contralateral side (Fig. 5). This lesion showed strong contrast enhancement (Fig. 6). The fine needle aspiration biopsy of this lesion identified scattered cells and small groups of plasma cells (Fig. 7).

The patient was initially treated with dexamethasone and intravenous infusion of $20 \%$ mannitol. Due to the extensiveness of the local disease and direct proximity of the sagittal sinus, neurosurgical treatment was not introduced. The conservative treatment was continued and she was admitted to the Department of Radiotherapy, where she received palliative brain irradiation, 20 Gray in 4 fractions. Despite a slight decrease in the tumour size and a fall in the plasma gamma globulin level, the clinical symptoms of paresis increased. Thalidomide ( $200 \mathrm{mg} /$ day) was started, but the patient died one month after the radiotherapy. 


\section{Discussion}

Neurological presentation occurs rarely in the course of multiple myeloma. Only individual case reports and small series described such symptomatology, including cranial nerve palsy [8], visual loss [6] or motor seizures [13]. The apoplectic symptoms are even more uncommon in MM. Stroke is defined as an abrupt onset of focal or global neurological symptoms caused by ischemia or haemorrhage. By convention, these symptoms must continue for more than 24 hours, otherwise such an episode is referred to as a transient ischemic attack (TIA) [7].

Ischemic stroke is a consequence of impaired blood supply to a certain area of the brain while haemorrhage is caused by bleeding outside the injured vessel within the CNS [7]. Most examples of an ischemic stroke result from atherosclerosis or thromboembolic events in an arterial vessel [7].

Haematological conditions are an unusual cause of ischemic stroke accounting for approximately $1 \%$ of first-time stroke patients [1,2]. They include disturbances of hemostasis, e.g. increased thrombogenic activity of the blood plasma, decreased performance of antithrombotic mechanisms, abnormalities of platelet function, anaemias, (sickle cell anaemia and thalassemias), as well as an increased blood viscosity as occurs in polycythemia or hyperviscosity syndrome [7].

Hyperviscosity syndrome is a clinical entity caused by an increased resistance to blood flow, frequently caused by hypergammaglobulinemia that is monoclonal in the majority of cases. Hyperviscosity syndrome occurring in plasma cell dyscrasias is most frequent in Waldenstrom's macroglobulinemia. Hyperviscosity syndrome may present with constitutional symptoms, bleeding, ocular, neurological and cardiovascular manifestations [9]. Neurological symptoms include headaches, vertigo, tinnitus, difficulty in concentrating, impaired hearing, ataxia and somnolence. Stroke is very uncommon [9].

In the literature, we found 3 patients with multiple myeloma that presented with stroke in the course of disease. This clinical appearance most probably depends on the hyperviscosity syndrome as all these patients had highly increased serum levels of gamma globulin $[2,4,12]$. One patient with MM was reported as suffering from an ischemic infarct. Low serum levels of monoclonal protein and presence of other risk factors (hypertension and history of TIA) did not in- dicate $M M$ as the main causative factor of this ischemic episode [1].

An additional pathogenic factor of ischemic stroke is a thromboembolic event, but as of now not a single case has been suggested in MM patients.

Arterial hypertension is the most frequent cause of intracranial haemorrhage. It is responsible for about $50 \%$ of cases. Intracranial bleeding occurs in approximately $8 \%$ of cases with primary and metastatic tumours. Glioblastoma predominates among primary tumours affected by bleeding, whereas metastases of bronchogenic carcinoma, choriocarcinoma, melanoma and renal cell carcinoma are the most common sources of intracranial haemorrhage in the secondary neoplasms of the CNS [7].

As multiple myeloma involvement of the CNS is rare [13], intracranial haemorrhage of the tumour is an extraordinary event in clinical practice. Only one case report of a 57-year-old male can be found in the literature. The patient presented with a strong headache, right-sided hemiparesis, confusion and dysphasia due to haemorrhage of the plasma cell tumour. The tumour involved the left temporal lobe, dura, sphenoid ridge and the squamous portion of the temporal lobe [3].

Another potential pathomechanism of stroke in patients with $M M$ is subdural hematoma. Although it is usually dependent on head trauma, such a relationship was not established in any of the three patients with $M M$ and subdural hematoma $[1,10,11]$. Additionally, a similar presentation was described in a patient with plasma cell leukaemia [10]. Thrombocytopenia and hyperviscosity syndrome were suggested as a potential pathogenic cause in this case.

Stroke-like symptoms (paresis and aphasia) may also result from direct local expansion of the neoplastic tissue in the brain. Our two patients seemingly presented with such a pathomechanism of their symptoms. We have not successfully retrieved similar cases of MM patients, although such a presentation may occur in primary and metastatic tumours of the CNS [5].

Our two cases are unique as their clinical presentation was seemingly dependent on direct compression of the brain tissue by tumour mass. This seems more probable since we did not observe any sign of bleeding, hypoxia or infarction within the skull cavity. The appearance of the clinical symptoms may also result from a relatively rapid growth of the MM in our cases. In the literature, the symptoms of stroke were usually secondary to the impaired blood supply due to changed rheological 
properties of the blood [2,4,12], observed regularly in the course of $\mathrm{MM}$, or secondary to the bleeding into the skull cavity $[1,3,10]$ so they were not attributed to the growth properties of the tumour cells.

\section{Conclusions}

We described two very unusual presentations of MM patients with stroke symptomatology secondary to mass effect on brain tissue.

Although symptoms originating from the CNS are infrequent in MM patients, stroke symptomatology can be observed as MM involvement of the CNS and occasionally it can be found as the initial manifestation.

\section{Acknowledgments}

This study was supported from the grant No. ST-95 from the Medical University of Gdańsk.

\section{References}

1. Arboix A, Besses C. Cerebrovascular disease as the initial clinical presentation of haematological disorders. Eur Neurol 1997; 37: 207-211.

2. Bogousslavsky J, Van Melle G, Regli F. The Lausanne Stroke Registry: analysis of 1,000 consecutive patients with first stroke. Stroke 1988; 19: 1083-1092.

3. Crowley RW, Sansur CA, Sheehan JP, Mandell JW, Kassell NF, Dumont AS. Intracranial plasmacytoma with apoplectic presentation and spontaneous intracerebral hemorrhage: Case report and review of the literature. Clin Neurol Neurosurg 2010; 112: 172-175.

4. Fagniez O, Tertian G, Dreyfus M, Ducreux D, Adams D, Denier C. Hematological disorders related cerebral infarctions are mostly multifocal. J Neurol Sci 2011; 15: 87-92.

5. Fisher M, Recht LD. Brain tumor presenting as an acute pure motor hemiparesis. Stroke 1989; 20: 288-291.

6. Hogan MC, Lee A, Solberg LA. Unusual presentation of multiple myeloma with unilateral visual loss and numb chin syndrome in a young adult. Am J Hematol 2002; 70: 55-59.

7. Kalimo H, Kaste M, Haltia M. Vascular diseases. In: Greenpield's Neuropathology. Graham DI, Lantos PL (eds.). Arnold Publishers, New York 2002.

8. Kashyap R, Kumar R, Kumar S. Cranial nerve palsy in multiple myeloma and solitary plasmacytoma. Asia Pac J Clin Oncol 2010; 6: 251-255.

9. Mehta J, Singhal S. Hyperviscosity syndrome in plasma cell dyscrasias. Semin Thromb Hemost 2003; 29: 467-471.

10. Minette SE, Kimmel DW. Subdural hematoma in patients with systemic cancer. Mayo Clin Proc 1989; 64: 637-642.

11. Mitsos A, Georgakoulias N, Jenkins A. Intracranial plasmacytoma presenting as chronic subdural haematoma. Br J Neurosurg 2004; 18: 647-649.
12. Park MS, Kim BC, Kim IK, Lee SH, Choi SM, Kim MK, Lee SS, Cho KH. Cerebral infarction in IgG multiple myeloma with hyperviscosity. J Korean Med Sci 2005; 20: 699-701.

13. Wavre A, Baur AS, Betz M, Mühlematter D, Jotterand M, Zaman K, Ketterer N. Case study of intracerebral plasmacytoma as an initial presentation of multiple myeloma. Neuro Oncol 2007; 9: 370-372. 